\title{
Redox Proteomics
}

\author{
D. Allan Butterfield ${ }^{1}$ and Isabella Dalle-Donne ${ }^{2}$
}

\begin{abstract}
Proteins are major targets of reactive oxygen and nitrogen species (ROS/RNS) and numerous post-translational, reversible or irreversible modifications have been characterized, which may lead to a change in the structure and/or function of the oxidized protein. Redox proteomics is an increasingly emerging branch of proteomics aimed at identifying and quantifying redox-based changes within the proteome both in redox signaling and under oxidative stress conditions. Correlation between protein oxidation and human disease is widely accepted, although elucidating cause and effect remains a challenge. Increasing biomedical data have provided compelling evidences for the involvement of perturbations in redox homeostasis in a large number of pathophysiological conditions and aging. Research toward a better understanding of the molecular mechanisms of a disease together with identification of specific targets of oxidative damage is urgently required. This is the power and potential of redox proteomics. In the last few years, combined proteomics, mass spectrometry (MS), and affinity chemistry-based methodologies have contributed in a significant way to provide a better understanding of protein oxidative modifications occurring in various biological specimens under different physiological and pathological conditions. Hence, this Forum on Redox Proteomics is timely. Original and review articles are presented on various subjects ranging from redox proteomics studies of oxidatively modified brain proteins in Alzheimer disease and animal models of Alzheimer and Parkinson disease, to potential new biomarker discovery paradigm for human disease, to chronic kidney disease, to protein nitration in aging and age-related neurodegenerative disorders, electrophile-responsive proteomes of special relevance to diseases involving mitochondrial alterations, to cardiovascular physiology and pathology. Antioxid. Redox Signal. 17, 1487-1489.
\end{abstract}

A MONG BIOMOLECULES, PROTEINS are major targets of reactive oxygen and nitrogen species (ROS/RNS) and numerous post-translational, reversible or irreversible modifications have been characterized, which may lead to a change in the structure and/or function of the oxidized protein. Oxidative protein modifications may result either from direct oxidation of amino acid residues or through the formation of reactive intermediates (i.e., reactive carbonyl species) generated during lipid peroxidation, which can react with the sulfhydryl group of cysteine, the $\varepsilon$-amino group of lysine or the imidazole group of histidine residues, forming advanced lipoxidation end products, or produced by the reaction of reducing sugars or their oxidation products with lysine residues of proteins, leading to the formation of advanced glycation end products.

Redox proteomics is an increasingly emerging branch of proteomics aimed at identifying and quantifying redox-based changes within the proteome both in redox signaling and under oxidative stress conditions (1-9). Proteins are unique among biological macromolecules in possessing elements (cysteine, methionine, and selenocysteine) that undergo reversible redox reactions as part of their normal function, which support dynamic regulation of structure and function. At the same time, they also can be important molecular signposts of irreversible oxidative damage at various amino acids under different pathophysiological conditions, which may contribute to establishing a relationship between pathological hallmarks of disease and protein structural/ functional abnormalities.

Alterations in the cellular redox status or in the levels of specific ROS and/or RNS are most often sensed by proteins with redox-reactive Cys residues, whose thiol oxidation states exert control over the activity and function of the proteins. Cys residues are thus the typical targets of redox modification, as they can adopt multiple oxidation states and a particular cysteine residue may be differentially modified in response to diverse stimuli.

The burgeoning field of redox proteomics $(1,6)$ has allowed a global perspective on the extent of protein oxidation in both normal and disease-affected cells. The correlates between

\footnotetext{
${ }^{1}$ Department of Chemistry, Center of Membrane Sciences, and Sanders-Brown Center on Aging, University of Kentucky, Lexington, Kentucky.

${ }^{2}$ Department of Life Sciences, University of Milan, Milan, Italy.
} 
protein oxidation and human disease are widely appreciated, although unraveling cause and effect remains a challenge. Increasing biomedical data have provided compelling evidences for the involvement of redox homeostasis perturbations in a large number of pathophysiological conditions, including type 2 diabetes mellitus, cardiovascular disease, atherosclerosis, stroke, many types of cancer, and neurodegenerative diseases such as Alzheimer's disease, mild cognitive impairment, amyotrophic lateral sclerosis, and Parkinson's disease $(1,6)$. Oxidative/nitrosative stress-and the resulting damage to the major classes of macromolecules in cells, mainly to proteins and lipids-is also involved in physiological aging and degenerative processes occurring in age-related diseases (1-9). However, definitive evidence for the link between oxidative damage and either the primary or secondary pathophysiological mechanisms of multiple acute and chronic human diseases has often been lacking because of recognized shortcomings with methods available to assess oxidative stress status and the resulting oxidative damage in vivo in humans.

In the last few years, combined proteomics, mass spectrometry (MS), and affinity chemistry-based methodologies have contributed in a significant way to provide a better understanding of protein oxidative modifications occurring in various biological specimens under different physiological and pathological conditions (e.g., 1-9). Indeed, redox proteomics results obtained in different human diseases and animal models thereof may provide new insights into the main mechanisms involved in the pathogenesis and progression of oxidative stress-related diseases, such as neurodegenerative disorders and cardiovascular disease.

The study of the role of ROS/RNS and oxidative stress in disease and aging involves the measurement of biomarkers of oxidative damage that reflect damage induced from an attack by specific ROS/RNS or secondary by-products of oxidative stress. In fact, although some ROS/RNS can be directly detected, currently available methods are not generally applicable to clinical examination due to the chemical instability of most ROS/RNS, which are too reactive and/or have a too short half-life. Therefore, such molecules cannot be isolated or measured directly in cells, tissues, and body fluids. In contrast, molecular products formed from the reaction of ROS/ RNS with various biomolecules are generally more stable than ROS/RNS themselves. As a consequence, in most cases, production of ROS/RNS has commonly been inferred by measuring stable metabolites and/or their oxidation target products, including stable markers of oxidative protein damage such as nitrotyrosine, dityrosine, and protein carbonyls (1-9).

Research toward a better understanding of the molecular mechanisms of a disease together with identification of specific targets of oxidative damage is urgently required. Concurrently, there is an overwhelming, urgent need to validate more specific oxidative damage biomarkers that can be easily measured in human biological fluids, to overcome limitations of invasive monitoring $(1,6)$. A few biomarkers of oxidative stress/damage are validated and commonly used. However, limited human data are available on the levels of specific biomarkers of protein damage in controlled clinical studies. Consequently, at present, it is unknown if some oxidative stress-induced protein modifications may be an important tool in clinical trials as biomarkers of oxidative stress and, more importantly, may have diagnostic (particularly early and possibly presymptomatic) and therapeutic uses. Current research progress on the detailed measurement of oxidative damage to proteins by different redox proteomics and MS technologies suggests that many more biomarkers are likely to be developed and clearly validated in the near future.

In contrast to measurement of individual biomarkers, proteomic analysis presents the opportunity to identify a panel of complementary biomarkers that can give extensive information regarding a disease $(1,6)$. Such biomarkers can be considered as a panel of up- and downregulated proteins with altered post-translational modifications, which differ in disease and normal state. Of course, although it is likely that some future results may ultimately show that changes in ROS/RNS concentration and oxidative protein damage in some situations are merely epiphenomena, it is also likely that future research development in redox proteomics will be able to provide the biomedical research community with new tools to assess the significance of oxidative protein modifications and, indirectly, of ROS/RNS, in biological systems. Progress in the field of redox proteomics should advance our knowledge of regulatory mechanisms that involve oxidation of proteins, especially at cysteine residues, and lead to a better understanding of oxidative biochemistry in health and disease. Thus, redox proteomics promises a significant contribution in the field of molecular biology and biomedical research, especially in the elucidation of redox-dependent and ROS/RNS-dependent signaling mechanisms and in the discovery of diagnostic biomarkers enabling early diagnosis of various oxidative stress-related human diseases. We believe that redox proteomics will contribute to broaden our knowledge concerning potential biomarkers for disease diagnosis and may also provide insight into damaged metabolic networks and potential targets for modulation of disease progression by means of drug therapy. Indeed, redox proteomics will plausibly play a pivotal role also in the search for new therapeutic targets and their validation, and in the design and testing of new drugs against those pathologies related to altered redox homeostasis.

In this Forum on Redox Proteomics, applications of these methods to animal models of Parkinson and Alzheimer disease are given in the original articles by Allan Butterfield et al. The article regarding mutant LRRK2 and mutant tau in a Caenorhabditis elegans model of Parkinson disease reveals that alterations in autophagy are critical to pathology relevant to Parkinson disease. The article relevant to Alzheimer disease involves proteomics studies of brain from a mouse with three mutations in amyloid precursor protein, from which the neurotoxic peptide, amyloid $\beta$-peptide [1-42] is obtained. The results demonstrate that replacement of the single methionine residue in amyloid $\beta$-peptide, previously shown to be critical to in vivo oxidative stress in brain, by leucine, leads to key brain proteins no longer having altered levels; by Marzia Perluigi et al. for analysis of 4-hydroxy-2-nonenal modified proteins in neurodegenerative disorders showing that lipid peroxidation is a seminal event in brain in numerous neurodegenerative disorders that affects key proteins related to the pathology and clinical presentation of each disorder; by Isabella Dalle-Donne et al. for analysis of albumin redox modifications occurring in various human pathophysiological conditions, evidencing that, to some extent, albumin oxidation is correlated to organ dysfunction. The review article by 
David Sheehan et al. describes proteomics and redox proteomics studies dedicated to chronic kidney disease and related conditions, both in humans and animal models, and suggests that redox proteomics may provide an underexploited tool kit for identification of novel biomarkers in this commonly-occurring pathology. The review article by Maria Feeney and Christian Schöneich focuses on recent progress made by means of proteomics studies in characterizing the effects of specific protein tyrosine oxidative and nitrative modifications on structure, function, aggregation, and pathogenesis, with an emphasis on implications for aging and agerelated diseases. In their review article, Victor Darley-Usmar et al. highlight the innovative concept of electrophile responsive proteomes (ERPs), that is, the post-translational modification of a cell sub-proteome at nucleophilic amino-acid residues by electrophilic reactive lipid species originating from lipid peroxidation. They discuss new concepts through which the oxylipidome modulates biological functions through the formation of ERPs in cells, thus integrating lipidomics and proteomics with cellular functions, and describe, in particular, ERPs that modulate antioxidant and antiinflammatory protective pathways, including the activation of Keap1/Nrf2 and the promotion of cell death through interactions with mitochondria. The exhaustive review article by Markus Bachschmid et al. specifically addresses the formation of various redox and oxidative stress proteomes, that is, signaling pathways and proteins sensitive to different reversible and irreversible oxidative post-translational modifications, in the cardiovascular system and demonstrates the relationship of these to animal pathology and biomarker identification.

This Forum on Redox Proteomics illuminates the power of these methods to better understand human health and physiology. In the future, we believe redox proteomics will continue to make significant contributions to new insights into physiology and disease.

\section{Acknowledgments}

This work was supported by grants from NIH to D.A.B.

\section{References}

1. Butterfield DA, Perluigi M, Reed T, Muharib T, Hughes C, Robinson R, and Sultana R. Redox proteomics in selected neurodegenerative disorders. From its infancy to future applications. Antioxid Redox Signal 17: 1610-1655, 2012.

2. Butterfield DA, Poon HF, St. Clair D, Keller JN, Pierce WM, Klein JB, and Markesbery WR. Redox proteomics identification of oxidatively modified hippocampal proteins in mild cognitive impairment: insights into the development of Alzheimer's disease. Neurobiol Dis 22: 223-232, 2006.
3. Butterfield DA, Reed T, Newman SF, and Sultana R. Roles of amyloid $\beta$-peptide-associated oxidative stress and brain protein modifications in the pathogenesis of Alzheimer's disease and mild cognitive impairment. Free Radic Biol Med 43: 658677, 2007.

4. Butterfield DA and Sultana R. Redox proteomics identification of oxidatively modified brain proteins in Alzheimer's disease and mild cognitive impairment: insights into the progression of this dementing disorder. J Alzheimers Dis 12: 61-72, 2007.

5. Dalle-Donne I, Rossi R, Colombo G, Giustarini D, and Milzani A. Protein S-glutathionylation: a regulatory device from bacteria to humans. Trends Biochem Sci 34: 85-96, 2009.

6. Dalle-Donne I, Scaloni A, and Butterfield DA. (Eds). Redox Proteomics: From Protein Modifications to Cellular Dysfunction and Disease. Hoboken: John Wiley \& Sons, Inc., 2006, p. 944.

7. Dalle-Donne I, Scaloni A, Giustarini D, Cavarra E, Tell G, Lungarella G, Colombo R, Rossi R, and Milzani A. Proteins as biomarkers of oxidative/nitrosative stress in diseases: the contribution of redox proteomics. Mass Spectrom Rev 24: 5599, 2005.

8. Giustarini D, Dalle-Donne I, Tsikas D, and Rossi R. Oxidative stress and human diseases: origin, link, measurement, mechanisms, and biomarkers. Crit Rev Clin Lab Sci 46: 241281, 2009.

9. Sultana R and Butterfield DA. Identification of the oxidative stress proteome in the brain. Free Radic Biol Med 50: 487-494, 2011.

$$
\begin{array}{r}
\text { Address correspondence to: } \\
\text { Prof. D. Allan Butterfield } \\
\text { Department of Chemistry } \\
\text { Center of Membrane Sciences } \\
\text { and Sanders-Brown Center on Aging } \\
\text { University of Kentucky } \\
121 \text { Chem-Phys Bldg } \\
\text { Lexington, KY } 40506 \\
\text { E-mail: dabcns@uky.edu }
\end{array}
$$

Date of first submission to ARS Central, June 6, 2012; date of acceptance, June 7, 2012.

$\begin{aligned} & \text { Abbreviations Used } \\ \mathrm{ERPs} & =\text { electrophile responsive proteomes } \\ \mathrm{MS} & =\text { mass spectrometry } \\ \mathrm{RNS} & =\text { reactive nitrogen species } \\ \mathrm{ROS} & =\text { reactive oxygen species }\end{aligned}$

